\title{
Age dependence of plasma phospholipid fatty acid levels: potential role of linoleic acid in the age-associated increase in docosahexaenoic acid and eicosapentaenoic acid concentrations
}

\author{
Renate H. M. de Groot ${ }^{1}$, Martin P. J. van Boxtel ${ }^{2}$, Olga J. G. Schiepers ${ }^{2}$, Gerard Hornstra ${ }^{3}$ and Jelle Jolles ${ }^{4}$ \\ ${ }^{1}$ Department of Psychiatry and Neuropsychology, Centre for Brain and Learning and School for Mental Health and Neuroscience \\ (MHeNS), Maastricht University, Maastricht, The Netherlands \\ ${ }^{2}$ Department of Psychiatry and Neuropsychology, School for Mental Health and Neuroscience (MHeNS), Maastricht University, \\ Maastricht, The Netherlands \\ ${ }^{3}$ Nutrition and Toxicology Research Institute Maastricht (NUTRIM), Maastricht University, Maastricht, The Netherlands \\ ${ }^{4}$ Faculty of Psychology and Education, Centre for Brain and Learning and AZIRE Research Institute, VU University Amsterdam, \\ Amsterdam, The Netherlands
}

(Received 19 November 2008 - Revised 19 March 2009 - Accepted 25 March 2009 - First published online 30 April 2009)

\begin{abstract}
Limited information is available with respect to the association between age and the plasma phospholipid fatty acid profile. Therefore we investigated the association between plasma phospholipid fatty acid status and age after correction for sex, smoking, alcohol use, BMI and fish intake. Plasma phospholipid fatty acid composition was measured and information on fish intake and other potential covariates was collected in 234 participants of the Maastricht Aging Study. The participants were healthy individuals of both sexes with an age range between 36 and 88 years. Hierarchical linear regression analyses were applied to study the relationship between age and fatty acid concentrations. After correction for fish consumption and other relevant covariates, a significant positive relationship was observed between age of the subjects and their plasma phospholipid concentrations of DHA $(22: 6 n-3, P=0.006)$ and EPA $(20: 5 n-3 ; P=0.001)$. Age contributed 2.3 and $3.9 \%$ to the amount of explained variance, respectively. The higher $n$-3 long-chain PUFA status at advanced age was confirmed by lower concentrations of their putative 'shortage marker' Osbond acid (ObA, $22: 5 n-6 ; P=0.022$ for the relationship with age after correction for covariates and fish intake, $\left.R^{2} 0.022\right)$. Concentrations of linoleic acid (LA; $18: 2 n-6)$ were negatively associated with age $\left(P<0 \cdot 001 ; R^{2} 0 \cdot 061\right)$. In conclusion, DHA and EPA concentrations appeared to be higher in older age groups, partly because of a higher fish intake and partly because of another age-associated mechanism, possibly involving the well-known competition with LA.
\end{abstract}

Fatty acids: Fish consumption: Ageing

In the Western world, CHD and Alzheimer's disease are among the most prominent complications of ageing. Nowadays, long-chain PUFA are increasingly recognised as compounds that may be associated with these diseases ${ }^{(1,2)}$. The limited information currently available with respect to the association between ageing and long-chain PUFA concentrations in plasma and erythrocytes is mainly restricted to the $n-3$ long-chain PUFA and suggests higher levels in older subjects ${ }^{(3-8)}$. However, in most of these studies the associations were adjusted incompletely, and most of them did not shine light on the relationship of $n-6$ fatty acids with age, thereby ignoring potential metabolic interactions between both essential fatty acid families. Moreover, the common understanding is that long-chain PUFA levels decrease with ageing, due to a lower fatty acid desaturation activity ${ }^{(9-11)}$. We therefore decided to study the potential age dependence of the entire plasma phospholipids fatty acid profile.
For this purpose we used a Dutch population sample, in which a relatively low fish consumption is the most important and almost the only source for $n-3$ long-chain PUFA intake.

\section{Methods and materials \\ Design and participants}

The present study was conducted as part of the 12-year follow-up assessment of the Maastricht Aging Study (MAAS). The MAAS is a prospective study in normal ageing adults varying in age from 24 to 81 years at baseline (i.e. 12 years previously) $^{(12,13)}$. The study was designed in order to investigate a broad range of objective cognitive measurements as a function of demographic as well as biological variables. The MAAS consists of four identical interleaved panel studies. The panels each consisted of $25 \%$ 
of the overall sample and were stratified for age, general ability level and sex.

From September 2005 onward the 12-year follow-up of the MAAS took place. Venous blood samples were collected in order to determine the fatty acid profile of plasma phospholipids. In addition, habitual fish consumption was assessed, as well as a number of other variables of potential importance in the relationship between fatty acid status and age.

All participants in the MAAS who were enrolled in the first panel were included in the present study. The study was approved by the local Medical Ethics Committee and written informed consent was obtained from each participant.

\section{Blood sampling, processing and fatty acid analyses}

Venous blood samples were collected in EDTA-containing tubes. After collection, plasma was separated from erythrocytes by centrifugation. Aliqouts of the plasma samples were divided over storage cups, which were closed tightly under $\mathrm{N}_{2}$ and stored at $-80^{\circ} \mathrm{C}$ until fatty acid analysis. Fatty acid profiles of phospholipids isolated from venous plasma were determined as described by $\mathrm{Al}$ et al. ${ }^{(14)}$ and Otto et al. ${ }^{(15)}$. After addition of an internal standard (1,2 dinonadecanoyl-sn-glycero-3-phosphocholine) plasma lipids were extracted by a modified Folch extraction method $^{(16)}$ and phospholipid fractions were isolated from the lipid extracts by using aminopropyl $(500 \mathrm{mg} / 4.0 \mathrm{ml})$ Extract-Clean columns ${ }^{(17)}$. Heptadecenoic acid was added to the samples to check for any carry-over of NEFA during the isolation of the phospholipids. The phospholipid fractions were hydrolysed and the fatty acid methylated with boron trifluoride in methanol ${ }^{(18)}$. The fatty acid composition of the phospholipids was then determined by capillary GC using a WCOT fused silica $(50 \mathrm{~m} \times 0.25 \mathrm{~mm}$ internal diameter $)$ CP-SIL 88 column (film thickness $0.2 \mu \mathrm{m}$ ) (Varian, Bergen op Zoom, the Netherlands), using $\mathrm{He}$ as the carrier gas. The injection and detection temperatures were $300^{\circ} \mathrm{C}$. The starting temperature of the column was $165^{\circ} \mathrm{C}$. After $10 \mathrm{~min}$, the temperature was increased $\left(5^{\circ} \mathrm{C} / \mathrm{min}\right)$ up to $190^{\circ} \mathrm{C}$, and kept constant for $15 \mathrm{~min}$. Finally the temperature was increased up to $230^{\circ} \mathrm{C}$ at the rate of $5^{\circ} \mathrm{C} / \mathrm{min}$.

Forty-two fatty acids were identified, but only the following selection will be reported: linoleic acid (LA; $18: 2 n-6) ; \gamma$-linolenic acid (18:3n-6); dihomo- $\gamma$-linolenic acid (20:3n-6); arachidonic acid (20:4n-6); adrenic acid (22:4n-6); Osbond acid (ObA; $22: 5 n-6) ; \alpha$-linolenic acid $(18: 3 n-3)$; stearidonic acid $(18: 4 n-3)$; eicosatetraenoic acid (20:4n-3); EPA (20:5n-3); docosapentaenoic acid (22:5n-3); DHA (22:6n-3). Relative fatty acid concentrations are reported as percentages of the total amount of phospholipid-associated fatty acids $(\%, w / w)$. In addition, the following fatty acid combinations are presented: sum of the SFA; sum of the MUFA.

The fatty acids of primary interest were arachidonic acid, adrenic acid, EPA, docosapentaenoic acid and DHA. The reason for this is that arachidonic acid, EPA and DHA are of importance in age-related diseases, such as $\mathrm{CVD}^{(1)}$ and Alzheimer's disease ${ }^{(2)}$, whereas adrenic acid is involved as a status parameter for DHA and docosapentaenoic acid is a major intermediate in the EPA-to-DHA conversion.

\section{Assessment of fish consumption}

Fish consumption was measured at 12-year follow-up by means of a newly validated short self-report questionnaire. Different fish types were categorised according to their respective DHA contents: low (fish fingers, prawns, pickled herring, cod, mussels, plaice, tuna and tilapia); intermediate (trout, raw herring, smoked eel, smoked salmon, canned salmon); high (smoked herring, herring in tomato sauce, mackerel, canned sardines, salmon) ${ }^{(19)}$. The frequency of consumption (never, once per month, two to three times per month, once per week, more often than once per week) was used to calculate the fish consumption score: $0,1,2,4$ and 8 for the 'low DHA' fish group; 0, 2, 4, 8 and 16 for the 'intermediate DHA' class; 0, 3, 6, 12 and 24 for the 'high DHA' category. Overall scores for fish consumption thus ranged from 0 to 48 and were validated against the EPA and DHA concentrations in plasma phospholipids of the participants (see the Statistical analyses section).

\section{Other measurements}

Sex, alcohol consumption, smoking and BMI $\left(\mathrm{kg} / \mathrm{m}^{2}\right)$ were treated as covariates, as these variables have been shown to be associated with fatty acid status ${ }^{(3,20,21)}$. Alcohol consumption, measured at 12-year follow-up by means of a short self-report questionnaire, was dichotomised into 'high' or 'low/none' intake categories, according to WHO standards ${ }^{(22)}$. 'High' was defined as the consumption of at least two (women) or three (men) alcoholic beverages (standard units, i.e. $8 \mathrm{~g}$ ethanol per drink) per $\mathrm{d}$ on average, which is considered to be harmful intake by the WHO. Data on smoking ('current smoker' yes/no) and BMI (continuous) were also collected at 12-year follow-up.

\section{Statistical analyses}

Descriptive analysis of the data included a check for outliers and for distribution of the dependent variables. Since the values of $\gamma$-linolenic acid, $\alpha$-linolenic acid, EPA and eicosatetraenoic acid were not distributed normally, a log transformation was applied for these variables before these variables entered the statistical analysis. Outliers with a Cook's distance larger than 0.04 were excluded from the dataset (maximum of three cases per analysis).

In order to ascertain whether the new short fish consumption questionnaire was a reliable measure of fatty acid intake, bivariate Pearson's correlation coefficients were calculated between total score on the questionnaire and the plasma phospholipid DHA and EPA concentrations.

To investigate whether our data were in accordance with findings by others, showing increases in $n-3$ long-chain PUFA with age, multiple linear regression analyses were performed, using age as the independent variable and the respective fatty acids of primary interest as dependent variables. Sex, smoking, alcohol and BMI were controlled for in all models. Next, this procedure was repeated for the other fatty acids.

Hierarchical linear regression analyses were performed to test to what extent differences in fish consumption explained the possible association between fatty acid concentration and 
age, and whether age explained any additional variance on top of fish consumption. To this end, fish consumption was entered in the first step of the analysis, the covariates (sex, smoking, alcohol, BMI) were entered in the second step, and finally age in the third step.

The $\alpha$ level of the analyses with the fatty acids of primary interest (arachidonic acid, adrenic acid, EPA, docosapentaenoic acid, DHA) was set at $P<0 \cdot 010(0 \cdot 05$ divided by 5) to correct for multiple testing. For the same reason, analyses of the other fatty acid data required adaptation of the $\alpha$ level to $P<0.003$ ( 0.05 divided by 15$)$.

All analyses were performed using the SPSS statistical package for Mac OS X (release 11.0.4; SPSS Inc., Chicago, IL, USA).

\section{Results}

\section{Participants}

The panel, from which the study sample was recruited, consisted at baseline (i.e. 12 years previously) of 470 participants. During the 12 years of follow-up, 169 participants left the study due to a variety of reasons (for example, deceased, serious illnesses, refusal). This implied a total of 301 eligible participants for the present study. Participation in the sidearm study that involved blood sampling was voluntary. Sixty participants refused to donate blood for fatty acid analyses. In another seven individuals, no information was available about their current smoking status and alcohol consumption. Therefore, 234 individuals participated in the present study.

Individuals who left the study were not significantly different from the remaining participants with respect to age, sex, educational level and BMI (results not shown). Except for those seven dropouts from whom information regarding alcohol consumption and smoking habits was lacking, dropouts did not differ from remaining participants with respect to alcohol intake and smoking habits either. The average age of the final population was 59.8 (SD 13.4; range 36.8-88.2) years and the average number of years of education was 11.4 (SD 3.1) years.
The research population consisted of 117 males and 117 females, with an average BMI of $26.4 \mathrm{~kg} / \mathrm{m}^{2}$. In the group $21 \%$ were smokers and $5.8 \%$ reported high alcohol consumption.

\section{Fatty acid composition}

In Table 1 the average plasma phospholipid fatty acid concentrations are shown. These are in accordance with concentrations found in other comparable populations in the Netherlands ${ }^{(23)}$.

\section{Validation of the fish consumption questionnaire}

Significant correlations were found between plasma phospholipid DHA and log-transformed EPA concentrations on the one hand and the fish consumption score as measured with the fish consumption questionnaire on the other hand ( $n$ 232, $r$ 0.459, $P<0.001$ for DHA; $r 0.277, P<0.001$ for $\log$-EPA). This demonstrates that this questionnaire provides a reliable measure of EPA and DHA intake from fish.

\section{Associations between fatty acid concentrations and age}

Table 1 also displays the results of the respective multiple regression analyses of the relationships between age on the one hand and the different fatty acids on the other hand, after correction for sex, smoking, alcohol and BMI. For the fatty acids of primary interest and after correction for multiple testing, positive associations with age were found for DHA $(P<0.001$; Fig. 1(a) $)$ and log-transformed EPA $(P<0.001$; Fig. 1(b)), indicating that in older individuals the plasma phospholipid concentrations of these fatty acids were higher. Other fatty acids of primary interest showed no significant associations with age. Of the remaining fatty acids, LA showed a significant association with age $(P<0 \cdot 001$; Fig. 1(c)), after correction for multiple testing. LA concentrations were lower in older individuals. In addition, ObA was negatively associated with age $(P=0.003$; Fig. $1(d))$.

Table 1. Mean plasma phospholipid fatty acid concentrations and the results of the different regression analyses, showing the overall amount of explained variance $\left(R^{2}\right)$ and the $P$ value of the overall model $(n 234) \dagger$

\begin{tabular}{|c|c|c|c|c|c|c|c|c|c|c|}
\hline \multirow[b]{2}{*}{ Fatty acid $(\% \mathrm{w} / \mathrm{w})$} & \multirow[b]{2}{*}{ Mean } & \multirow[b]{2}{*}{ SD } & \multirow[b]{2}{*}{ Constant } & \multicolumn{5}{|c|}{ Regression coefficient } & \multirow[b]{2}{*}{$R^{2}$} & \multirow[b]{2}{*}{$P$} \\
\hline & & & & Age & Sex & Smoking & Alcohol & BMI & & \\
\hline Linoleic acid & $21 \cdot 830$ & $2 \cdot 898$ & $30 \cdot 340$ & $-0.056^{\star \star \star}$ & $-1.084^{\star \star}$ & 0.137 & -0.880 & $-0.172^{\star \star \star}$ & 0.168 & 0.000 \\
\hline$\gamma$-Linolenic acid & 0.079 & 0.042 & -1.398 & 0.000 & 0.027 & 0.024 & $0.174^{\star}$ & $0.008^{\star}$ & 0.054 & 0.027 \\
\hline Dihomo- $\gamma$-linolenic acid & $3 \cdot 183$ & 0.736 & $2 \cdot 243$ & $-0.010^{\star \star}$ & -0.032 & 0.116 & $-0.544^{\star \star}$ & $0.575^{\star \star \star}$ & 0.180 & 0.000 \\
\hline Arachidonic acid & $9 \cdot 611$ & 2.090 & $6 \cdot 724$ & 0.012 & -0.036 & -0.079 & 0.980 & $0.082^{\star *}$ & 0.046 & 0.057 \\
\hline Adrenic acid & 0.288 & 0.078 & 0.323 & -0.001 & $-0.033^{\star *}$ & 0.008 & 0.012 & 0.000 & 0.054 & 0.028 \\
\hline Osbond acid & 0.225 & 0.089 & 0.240 & $-0.001^{\star \star}$ & 0.000 & -0.011 & -0.013 & 0.002 & 0.051 & 0.036 \\
\hline$\alpha$-Linolenic acid & 0.175 & 0.059 & -0.746 & 0.000 & $0.039^{*}$ & -0.003 & -0.042 & -0.002 & 0.025 & 0.328 \\
\hline Stearidonic acid & 0.113 & 0.045 & 0.145 & 0.000 & -0.009 & 0.000 & -0.016 & 0.000 & 0.027 & 0.283 \\
\hline Eicosatetraenoic acid & 0.122 & 0.059 & $-1 \cdot 186$ & -0.001 & $0.058^{*}$ & 0.006 & -0.078 & $0.010^{\star *}$ & 0.043 & 0.011 \\
\hline EPA & 0.987 & 0.488 & -0.459 & $0.004^{\star \star \star}$ & $0.077^{\star \star}$ & $-0.066^{\star}$ & 0.025 & 0.006 & 0.126 & 0.000 \\
\hline Docosapentaenoic acid & 0.924 & 0.184 & 0.873 & 0.000 & -0.017 & $-0.058^{*}$ & -0.022 & 0.002 & 0.027 & 0.286 \\
\hline DHA & 3.446 & 1.037 & 2.085 & $0.019^{* \star *}$ & 0.086 & -0.296 & -0.355 & 0.011 & 0.087 & 0.001 \\
\hline$\Sigma$ SFA & 44.923 & 1.476 & $42 \cdot 244$ & $0.014^{*}$ & $0.435^{\star \star}$ & 0.052 & -0.008 & $0.057^{* *}$ & 0.091 & 0.001 \\
\hline$\Sigma$ MUFA & $12 \cdot 791$ & 1.384 & 12.833 & 0.008 & $0.357^{\star}$ & 0.106 & $0.786^{\star}$ & -0.031 & 0.058 & 0.018 \\
\hline
\end{tabular}

${ }^{\star} P<0.05,{ }^{\star *} P<0.01,{ }^{* \star *} P<0.001$.

†Age was a continuous variable; sex was coded 0 for men and 1 for women; smoking was coded 0 for non-smokers and 1 for smokers; alcohol was coded 0 for low alcohol consumption and 1 for high alcohol consumption; BMI was a continuous variable. For $\gamma$-linolenic acid, $\alpha$-linolenic acid, EPA and eicosatetraenoic acid the results of the regression analyses on the log-transformed data are shown. 

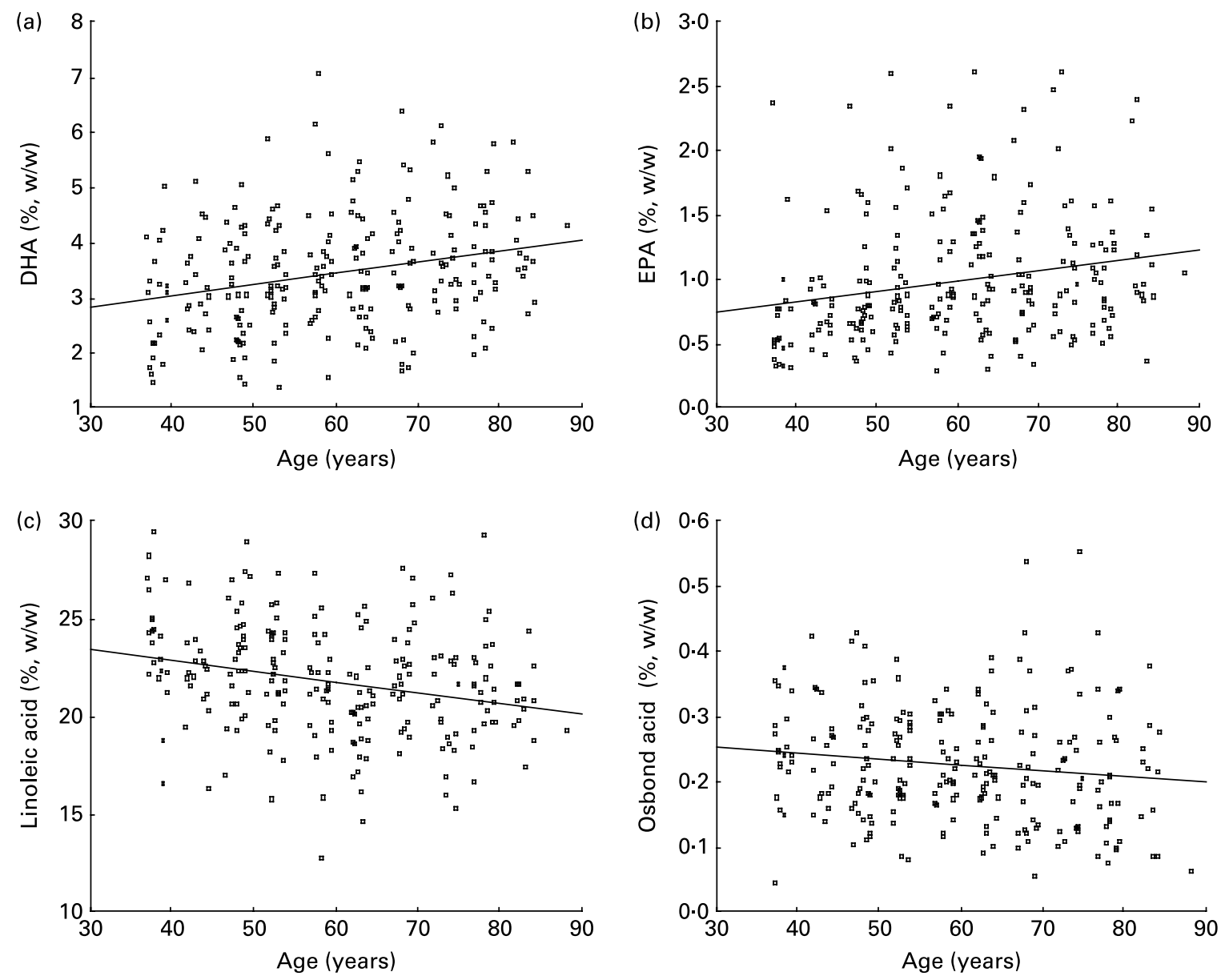

Fig. 1. Scatter plots of the relationship between, respectively, DHA (a), EPA (b), linoleic acid (c) and Osbond acid (d) and age. ( - ), Regression lines.

\section{Associations between fatty acid status and age after correction} for fish consumption

Hierarchical linear regression analyses with fish consumption in the first step, the other possible confounders (sex, smoking, alcohol and BMI) in the second step, and age in the third step (see Table 2) showed that after correction for fish consumption and other covariates, age explained an additional $2.3 \% \quad\left(R_{\text {Step } 3}^{2}-R_{\text {Step } 2}^{2}=0.336-0.313\right)$ of the explained variance in DHA on top of fish consumption. For EPA the contribution of age was $3.9 \%$ and for ObA $2.2 \%$. Thus, in the older age group the DHA and EPA concentrations were significantly higher, independent of fish intake, whereas ObA was significantly lower.

In case of LA, fish consumption did not significantly contribute to the amount of explained variance, whereas age contributed $6 \cdot 1 \%$.

\section{Discussion}

The goal of the present study was to investigate the age dependence of the entire plasma phospholipid profile. There was specific attention paid to EPA and DHA concentrations after correction for fish intake, as these fatty acids seem to play a role in some of the most common age-related diseases, i.e. CHD and Alzheimer's disease ${ }^{(1,2)}$.
In order to do so, the fatty acid concentrations of 234 male and female subjects with an age range of 36 to 88 years were determined and their fish consumptions assessed.

We found that after correction for sex, smoking, alcohol intake, BMI and multiple testing, the concentrations of DHA and EPA were positively associated with age, whereas the opposite was found for ObA. Also, LA was negatively associated with age (Table 1).

The findings with respect to EPA and DHA are largely in accordance with previous studies. Crowe, Dewailly and colleagues showed in Canadian and New Zealand populations that DHA and EPA concentrations are higher in plasma or serum phospholipids of elderly individuals compared with younger individuals ${ }^{(3-6)}$. Also, the results of Sands et al. ${ }^{(8)}$ and Itomura et al. ${ }^{(7)}$ are also fully in line with the present results. On the other hand, Babin et al. did not find significant EPA and DHA differences in plasma total lipids, TAG, cholesteryl esters and phospholipids, and in erythrocyte total lipids of 200 healthy elderly women over 75 years of age, compared with fifty young female volunteers aged $20-48$ years $^{(9)}$.

Studies on the relationship between fatty acid concentrations in tissues and age are scarce and results so far are not entirely consistent. Tavendale et al. investigated the adipose tissue fatty acid composition of 4114 individuals aged 40-59 years of the Scottish Heart Health Study and 
Table 2. Results of regression analyses of fish consumption (step 1), fish consumption and other possible confounders (step 2) and fish consumption, other possible confounders and age (step 3), showing the regression coefficients $B$ with their standard errors, the associated $P$ values and the total proportions of variance explained $\left(R^{2}\right) \dagger$

\begin{tabular}{|c|c|c|c|c|c|c|c|c|c|c|c|c|}
\hline & \multicolumn{4}{|c|}{ Step 1} & \multicolumn{4}{|c|}{ Step 2} & \multicolumn{4}{|c|}{ Step 3} \\
\hline & $B$ & SE & $P$ & $R^{2}$ & $B$ & SE & $P$ & $R^{2}$ & $B$ & SE & $P$ & $R^{2}$ \\
\hline \multicolumn{13}{|l|}{$\mathrm{DHA}$} \\
\hline Fish & 0.088 & 0.009 & 0.000 & & 0.087 & 0.009 & 0.000 & & 0.083 & 0.009 & 0.000 & \\
\hline Sex & & & & & 0.147 & 0.115 & 0.203 & & 0.150 & 0.114 & 0.188 & \\
\hline Smoking & & & & & -0.277 & 0.139 & 0.047 & & -0.237 & 0.137 & 0.086 & \\
\hline Alcohol & & & & & -0.301 & 0.238 & 0.206 & & -0.310 & 0.234 & 0.187 & \\
\hline BMI & & & & & 0.014 & 0.013 & 0.291 & & 0.012 & 0.013 & 0.338 & \\
\hline Age & & & & & & & & & 0.012 & 0.004 & 0.006 & \\
\hline & & & & 0.289 & & & & 0.313 & & & & 0.336 \\
\hline \multicolumn{13}{|l|}{ EPA } \\
\hline Fish & 0.008 & 0.002 & 0.000 & & 0.008 & 0.002 & 0.000 & & 0.007 & 0.002 & 0.000 & \\
\hline Sex & & & & & 0.074 & 0.026 & 0.004 & & 0.075 & 0.025 & 0.004 & \\
\hline Smoking & & & & & -0.073 & 0.031 & 0.019 & & -0.061 & 0.030 & 0.045 & \\
\hline Alcohol & & & & & 0.036 & 0.055 & 0.516 & & 0.032 & 0.054 & 0.554 & \\
\hline BMI & & & & & 0.007 & 0.003 & 0.019 & & 0.006 & 0.003 & 0.031 & \\
\hline Age & & & & & & & & & 0.003 & 0.001 & 0.001 & \\
\hline & & & & 0.076 & & & & 0.143 & & & & 0.182 \\
\hline \multicolumn{13}{|l|}{ Osbond acid } \\
\hline Fish & -0.003 & 0.001 & 0.001 & & -0.003 & 0.001 & 0.001 & & -0.002 & 0.001 & 0.004 & \\
\hline Sex & & & & & -0.007 & 0.011 & 0.519 & & -0.008 & 0.011 & 0.486 & \\
\hline Smoking & & & & & -0.001 & 0.013 & 0.914 & & -0.005 & 0.013 & 0.721 & \\
\hline Alcohol & & & & & -0.004 & 0.022 & 0.845 & & -0.004 & 0.022 & 0.873 & \\
\hline $\mathrm{BMI}$ & & & & & 0.002 & 0.001 & 0.132 & & 0.002 & 0.001 & 0.104 & \\
\hline Age & & & & & & & & & 0.001 & 0.000 & 0.022 & \\
\hline & & & & 0.047 & & & & 0.059 & & & & 0.081 \\
\hline \multicolumn{13}{|l|}{ Linoleic acid } \\
\hline Fish & -0.033 & 0.027 & 0.226 & & -0.039 & 0.026 & 0.141 & & -0.023 & 0.026 & 0.362 & \\
\hline Sex & & & & & -0.998 & 0.369 & 0.007 & & -1.049 & 0.357 & 0.004 & \\
\hline Smoking & & & & & 0.268 & 0.451 & 0.553 & & 0.103 & 0.438 & 0.815 & \\
\hline Alcohol & & & & & -1.321 & 0.851 & 0.122 & & -1.347 & 0.822 & 0.103 & \\
\hline BMI & & & & & -0.183 & 0.042 & 0.000 & & -0.175 & 0.040 & 0.000 & \\
\hline Age & & & & & & & & & -0.055 & 0.014 & 0.000 & \\
\hline & & & & 0.006 & & & & 0.116 & & & & 0.177 \\
\hline
\end{tabular}

†Age was a continuous variable; sex was coded 0 for men and 1 for women; smoking was coded 0 for non-smokers and 1 for smokers; alcohol was coded 0 for low alcohol consumption and 1 for high alcohol consumption; BMI was a continuous variable.

observed higher DHA levels (EPA concentrations were not reported) with advancing age ${ }^{(24)}$. This was confirmed in a later study ${ }^{(25)}$. Finally, Carver et al. studied the fatty acid composition of human brain at various ages and observed different age-related patterns for the various fatty acids, with no significant association for DHA (EPA was not reported) ${ }^{(26)}$. For plasma it now seems pretty well established, however, that ageing is associated with higher concentrations of $n-3$ long-chain PUFA.

A higher $n$-3 long-chain PUFA status at advanced age is supported by our observation that higher age is associated with lower concentrations of ObA, which is considered a biochemical marker of the functional DHA status ${ }^{(27,28)}$. Although this does not seem to hold under all circumstances ${ }^{(29)}$, the present study population is characterised by a strong negative relationship between DHA and ObA $(r-0.286 ; P<0.001)$, which supports the view that, at least in this particular group, $\mathrm{ObA}$ is a reliable indicator of the DHA status.

In general there is a strong association between fish consumption and the plasma concentrations of the $n-3$ long-chain PUFA ${ }^{(6,30,31)}$. This was also observed in the present study (for example, $r 0.459, P<0.001$ for the association between fish consumption and DHA). In addition, linear regression analysis showed a significant positive association between fish consumption and age ( $n$ 236; $r$ 0.157; $P=0.016$ ), demonstrating that fish intake was higher in the older participants. This finding of higher fish consumption in older age groups is in line with earlier reports on fish consumption ${ }^{(32-34)}$.

Whether a higher fish consumption explained the higher $n-3$ long-chain PUFA levels in elderly individuals, or whether additional age-dependent mechanisms contributed to the amount of explained variance, was also addressed in the present study. We showed that after correction for fish consumption and other covariates, age still contributed significantly to the model $(2.3 \%$ of explained variance in DHA, $3.9 \%$ in EPA, and $2.2 \%$ in $\mathrm{ObA}$ ). Others also concluded from their studies that correction for the intake of fish or n-3 fatty acids could not fully explain the apparent age dependence of plasma EPA and DHA concentrations ${ }^{(7-9,25)}$.

At this stage, we can only speculate about additional cause(s) of the higher DHA and EPA concentrations with increased age. A first explanation may include an increased intestinal fatty acid absorption with ageing. However, this does not explain why we observed the LA levels to be lower at higher age, a finding that was also reported by Tavendale et al. with respect to adipose tissue ${ }^{(24)}$. 
As an alternative, an altered fatty acid metabolism can be mentioned, for instance evident from an enhanced $\alpha$-linolenic acid-to-DHA conversion rate. However, on the basis of rat studies this does not seem very likely, as fatty acid desaturation seems to slow down upon ageing ${ }^{(10,11)}$ and also from the Babin et al. study a reduced synthesis of long-chain PUFA from their respective precursors upon ageing is suggested in $\operatorname{man}^{(9)}$. However, a stable-isotope study by Vermunt et al. ${ }^{(35)}$ suggested that endogenous DHA synthesis from $\alpha$-linolenic acid is not decreased at higher age, and may even be more effective.

A more likely explanation may be provided by our observation that plasma phospholipid LA concentrations are reduced at advanced age (Table 1). This confirms earlier findings by others, demonstrating lower LA concentrations in various plasma lipid fractions ${ }^{(9)}$ and adipose tissue ${ }^{(24,25,36)}$ of older as compared with younger women, and suggests a lower habitual LA consumption by older subjects ${ }^{(37)}$. It has been found repeatedly that LA inhibits the incorporation of $n$-3 long-chain PUFA in plasma phospholipids ${ }^{(38,39)}$, which may be the reason for the usually observed negative relationship in plasma phospholipids between LA and most long-chain PUFA concentrations. In the present study, these relationships also appeared to be negative and highly significant with correlation coefficients between -0.058 (for eicosatetraenoic acid; $20: 4 n-6$ ) and -0.393 (for EPA; for DHA, $r-0.277$ ). Interestingly, the correlation coefficients for the LA-DHA relationship were significantly higher in the oldest $(r-0 \cdot 015$; $P=0.916)$ as compared with the youngest quintile $(r-0.431$; $P=0.002)$. We therefore suggest that the fish intakeindependent mechanisms underlying the higher $n-3$ long-chain PUFA concentrations in plasma phospholipids at advanced age might include some competition between $n-3$ long-chain PUFA and LA. Further studies are required to test this hypothesis, and, if valid, to characterise the nature of this competition.

Besides its strengths such as the representative sample, and the correction for sex, smoking, alcohol intake, BMI and fish intake, the present study also has some limitations. The short self-report questionnaire to measure fish intake was specifically designed for the present study, based on earlier work, but has never been used in its current form. It was intended as a concise measure of habitual fish consumption, as only information was collected about the kind of fish that was consumed on a regular basis (based upon average DHA content) and the frequency of consumption. Portion size was not taken into account. There is a strong indication that our short questionnaire provided a reliable proxy of fish intake, as EPA and DHA concentrations in plasma phospholipids strongly correlated with fish intake. These correlations are comparable with or even better than correlations between other estimates for fish intake and plasma concentrations found in previous studies ${ }^{(40-42)}$.

As the present study was executed in a sample that was already followed up for 12 years for other purposes, selective attrition might be another potential drawback. This source of potential bias seems unlikely, however, as our data showed no differences in important baseline characteristics between dropouts and participants. Still, we cannot exclude a possible over- or underestimation of the observed associations due to this factor.
Although the contribution of age to the explained variance of the concentrations of the respective fatty acids was not large in an absolute sense, we do believe that the present results provide a strong indication that the DHA and EPA concentrations increase as part of the ageing process. The fact that we found an age effect in a relatively small sample ( $n$ 234) implies that it is a robust effect. It is generally accepted, for example, that smoking and alcohol consumption do influence fatty acid status ${ }^{(21)}$. Additional analyses (results not shown) in our sample showed that alcohol consumption added $0.05 \%$ to the amount of explained variance, whereas for smoking this was only $0.09 \%$. In this respect our finding of 2.3 and $3.9 \%$ explained variance for age is large and certainly a factor which should be considered in future research.

In conclusion, the present study showed that DHA and EPA concentrations were higher in older age groups, partly because of a higher fish intake and partly because of another age-associated mechanism, possibly involving competition with LA.

\section{Acknowledgements}

We would like to acknowledge the financial support of EFAMOL Ltd (Brackenholme, Selby, North Yorks, UK) for financial support (grant number CT05.4) and thank Dr Reto Muggli and Dr Peter Clough for their helpful advice.

All authors contributed substantially to the conception and design of the study. R. H. M. deG. was the principal investigator, and had major responsibility for protocol development, data analysis and writing of the manuscript. M. P. J. vB. was the supervisor of the MAAS study. He contributed to the statistical analyses and writing of the manuscript. O. J. G. S. also participated in reporting on the results of the study. G. H. contributed to the analytical protocol, interpretation of the fatty acid data and writing of the manuscript. J. J. was involved in the study design and the study management, and critically reviewed the manuscript.

There is no conflict of interest for any of the authors.

\section{References}

1. Lemaitre RN, King IB, Mozaffarian D, et al. (2003) n-3 Polyunsaturated fatty acids, fatal ischemic heart disease, and nonfatal myocardial infarction in older adults: the Cardiovascular Health Study. Am J Clin Nutr 77, 319-325.

2. Lim WS, Gammack JK \& Van Niekerk J, et al. (2006) Omega 3 fatty acid for the prevention of dementia. The Cochrane Database of Systematic Reviews 2006, issue 1, CD005379. http://www.mrw.interscience.wiley.com/cochrane/clsysrev/articl es/CD005379/frame.html

3. Crowe FL, Skeaff CM, Green TJ, et al. (2008) Serum n-3 longchain PUFA differ by sex and age in a population-based survey of New Zealand adolescents and adults. Br J Nutr 99, 168-174.

4. Dewailly E, Blanchet C, Gingras S, et al. (2002) Cardiovascular disease risk factors and $n-3$ fatty acid status in the adult population of James Bay Cree. Am J Clin Nutr 76, 85-92.

5. Dewailly E, Blanchet C, Lemieux S, et al. (2001) n-3 Fatty acids and cardiovascular disease risk factors among the Inuit of Nunavik. Am J Clin Nutr 74, 464-473. 
6. Dewailly EE, Blanchet C, Gingras S, et al. (2001) Relations between $n-3$ fatty acid status and cardiovascular disease risk factors among Quebecers. Am J Clin Nutr 74, 603-611.

7. Itomura M, Fujioka S, Hamazaki K, et al. (2008) Factors influencing EPA + DHA levels in red blood cells in Japan. In Vivo 22, 131-135.

8. Sands SA, Reid KJ, Windsor SL, et al. (2005) The impact of age, body mass index, and fish intake on the EPA and DHA content of human erythrocytes. Lipids 40, 343-347.

9. Babin F, Abderrazik M, Favier F, et al. (1999) Differences between polyunsaturated fatty acid status of non-institutionalised elderly women and younger controls: a bioconversion defect can be suspected. Eur J Clin Nutr 53, 591-596.

10. Biagi PL, Bordoni A, Hrelia S, et al. (1991) $\gamma$-Linolenic acid dietary supplementation can reverse the aging influence on rat liver microsome $\Delta 6$-desaturase activity. Biochim Biophys Acta 1083, 187-192.

11. Hrelia S, Bordoni A, Celadon M, et al. (1989) Age-related changes in linoleate and $\alpha$-linolenate desaturation by rat liver microsomes. Biochem Biophys Res Comm 163, 348-355.

12. Jolles J, Houx P, van Boxtel MP, et al. (1995) The Maastricht Aging Study; Determinants of Cognitive Aging. Maastricht: Neuropsych Publishers.

13. van Boxtel MP, Buntinx F, Houx PJ, et al. (1998) The relation between morbidity and cognitive performance in a normal aging population. J Gerontol A Biol Sci Med Sci 53, M147-M154.

14. Al MD, van Houwelingen AC, Kester AD, et al. (1995) Maternal essential fatty acid patterns during normal pregnancy and their relationship to the neonatal essential fatty acid status. Br J Nutr 74, 55-68.

15. Otto SJ, Houwelingen AC, Antal M, et al. (1997) Maternal and neonatal essential fatty acid status in phospholipids: an international comparative study. Eur J Clin Nutr 51, 232-242.

16. Bligh EG \& Dyer WJ (1959) A rapid method of total lipid extraction and purification. Can J Biochem Physiol 37, 911-917.

17. Kaluzny MA, Duncan LA, Merritt MV, et al. (1985) Rapid separation of lipid classes in high yield and purity using bonded phase columns. J Lipid Res 26, 135-140.

18. Morrison WR \& Smith LM (1964) Preparation of fatty acid methyl esters and dimethylacetals from lipids with boron fluoride-methanol. J Lipid Res 5, 600-608.

19. Stichting Nederlands Voedingsstoffenbestand (2003) NEVO Tabel 2003 (Dutch Food Composition Table 2003). Zeist, The Netherlands: NEVO.

20. Feart C, Jutand MA, Larrieu S, et al. (2007) Energy, macronutrient and fatty acid intake of French elderly community dwellers and association with socio-demographic characteristics: data from the Bordeaux sample of the Three-City Study. Br J Nutr 98, 1046-1057.

21. Simon JA, Fong J, Bernert JT Jr, et al. (1996) Relation of smoking and alcohol consumption to serum fatty acids. Am $J$ Epidemiol 144, 325-334.

22. World Health Organization (2000) International Guide for Monitoring Alcohol Consumption and Related Harm. Geneva: WHO.

23. Tiemeier H, van Tuijl HR, Hofman A, et al. (2003) Plasma fatty acid composition and depression are associated in the elderly: the Rotterdam Study. Am J Clin Nutr 78, 40-46.

24. Tavendale R, Lee AJ, Smith WC, et al. (1992) Adipose tissue fatty acids in Scottish men and women: results from the Scottish Heart Health Study. Atherosclerosis 94, 161-169.
25. Bolton-Smith C, Woodward M \& Tavendale R (1997) Evidence for age-related differences in the fatty acid composition of human adipose tissue, independent of diet. Eur J Clin Nutr 51, 619-624.

26. Carver JD, Benford VJ, Han B, et al. (2001) The relationship between age and the fatty acid composition of cerebral cortex and erythrocytes in human subjects. Brain Res Bull 56, 79-85.

27. Galli C, Trzeciak HI \& Paoletti R (1971) Effects of dietary fatty acids on the fatty acid composition of brain ethanolamine phosphoglyceride: reciprocal replacement of $n-6$ and $n-3$ polyunsaturated fatty acids. Biochim Biophys Acta 248, 449-454.

28. Neuringer M, Connor WE, Lin DS, et al. (1986) Biochemical and functional effects of prenatal and postnatal omega 3 fatty acid deficiency on retina and brain in rhesus monkeys. Proc Natl Acad Sci U S A 83, 4021-4025.

29. Innis SM, Vaghri Z \& King DJ (2004) n-6 Docosapentaenoic acid is not a predictor of low docosahexaenoic acid status in Canadian preschool children. Am J Clin Nutr 80, 768-773.

30. Anttolainen M, Valsta LM, Alfthan G, et al. (1996) Effect of extreme fish consumption on dietary and plasma antioxidant levels and fatty acid composition. Eur J Clin Nutr 50, 741-746.

31. van Houwelingen AC, Hornstra G, Kromhout D, et al. (1989) Habitual fish consumption, fatty acids of serum phospholipids and platelet function. Atherosclerosis 75, 157-165.

32. Myrland Ø, Trondsen T, Johnston RS, et al. (2000) Determinants of seafood consumption in Norway: lifestyle, revealed preferences, and barriers to consumption. Food Qual Prefer 11, $169-188$.

33. Olsen SO (2003) Understanding the relationship between age and seafood consumption: the mediating role of attitude, health involvement and convenience. Food Qual Prefer 14, 199-209.

34. Trondsen T, Scholderer J, Lund E, et al. (2003) Perceived barriers to consumption of fish among Norwegian women. Appetite 41, 301-314.

35. Vermunt SH, Mensink RP, Simonis AM, et al. (1999) Effects of age and dietary $n-3$ fatty acids on the metabolism of $\left[{ }^{13} \mathrm{C}\right]-\alpha$-linolenic acid. Lipids 34, Suppl., S127.

36. Wahle KW, McIntosh G, Duncan WR, et al. (1991) Concentrations of linoleic acid in adipose tissue differ with age in women but not men. Eur J Clin Nutr 45, 195-202.

37. Baylin A, Kabagambe EK, Siles X, et al. (2002) Adipose tissue biomarkers of fatty acid intake. Am J Clin Nutr 76, 750-757.

38. Cleland LG, James MJ, Neumann MA, et al. (1992) Linoleate inhibits EPA incorporation from dietary fish-oil supplements in human subjects. Am J Clin Nutr 55, 395-399.

39. Gronn M, Gorbitz C, Christensen E, et al. (1991) Dietary n-6 fatty acids inhibit the incorporation of dietary $n-3$ fatty acids in thrombocyte and serum phospholipids in humans: a controlled dietetic study. Scand J Clin Lab Invest 51, 255-263.

40. Kalmijn S, van Boxtel MP, Ocke M, et al. (2004) Dietary intake of fatty acids and fish in relation to cognitive performance at middle age. Neurology 62, 275-280.

41. Mina K, Fritschi L \& Knuiman M (2008) Do aggregates of multiple questions better capture overall fish consumption than summary questions? Public Health Nutr 11, 196-202.

42. Woods RK, Stoney RM, Ireland PD, et al. (2002) A valid food frequency questionnaire for measuring dietary fish intake. Asia Pacific J Clin Nutr 11, 56-61. 